\title{
Associação entre polimorfismo SLC6A3 3'UTR VNTR e a resposta ao tratamento da dependência de nicotina
}

\author{
Association between polymorphism SLC6A3 3'UTR VNTR \\ and response to treatment of nicotine dependence \\ Guilherme Rubino de Azevedo Focchi', Ismael Dale Cotrim Guerreiro da Silva², Sandra Scivoletto ${ }^{3}$
}

\section{RESUMO}

Objetivo: Avaliar a associação entre a resposta ao tratamento da dependência de nicotina com bupropiona e a presença do polimorfismo SLC6A3 3'UTR VNTR, localizado no gene que codifica o transportador dopaminérgico. Método: Foram acompanhados no Ambulatório de Tabagismo do Instituto de Psiquiatria da Faculdade de Medicina da USP 100 pacientes do sexo masculino com diagnóstico de dependência de nicotina, sem outras patologias. Todos receberam bupropiona até $300 \mathrm{mg}$ ao dia por 12 semanas, associada à terapia cognitivo-comportamental em grupo. A Escala de Fagerström foi aplicada no início e no final do tratamento, e avaliou-se a parada do uso de cigarros na última semana de tratamento e um mês após. Os pacientes tiveram $10 \mathrm{ml}$ de sangue colhidos e genotipados para a existência do polimorfismo SLC6A3 3'UTR VNTR. Resultados: Não foi encontrada associação entre cessação do uso de cigarro e presença do polimorfismo. Conclusão: São necessários mais estudos para avaliar se a presença do polimorfismo SLC6A3 3'UTR VNTR estaria relacionada à melhor resposta ao tratamento da dependência de nicotina.

\section{Palavras-chave}

Dependência, nicotina, abstinência, polimorfismo.

\section{Keywords}

Dependence, nicotine, withdrawal, polymorphism.

\begin{abstract}
Objective: To evaluate the association between response to treatment of nicotine dependence with bupropion and the presence of the polymorphism SLC6A3 3'UTR VNTR, in the gene that codifies the dopaminergic transporter. Method: A hundred patients were treated in the Nicotine Dependence Outpatient Clinic of the Institute of Psychiatry, University of São Paulo Medical School. All patients were male, diagnosed as nicotine dependents and had no other diseases. All received bupropion until $300 \mathrm{mg}$ a day for 12 weeks, combined with cognitive-behavioral group therapy. The Fagerström Scale was applied at the beginning and at the end of treatment. Cigarette cessation was evaluated in the last week of treatment and one month later. Patients had $10 \mathrm{ml}$ blood extracted and genotiped for SLC6A3 3'UTR VNTR polymorphism. Results: There was no association between cigarettes cessation and the presence of polymorphism. Conclusion: More studies are needed to assess whether the presence of polymorphism SLC6A3 3'UTR VNTR could be associated with a better response to treatment of nicotine dependence.
\end{abstract}

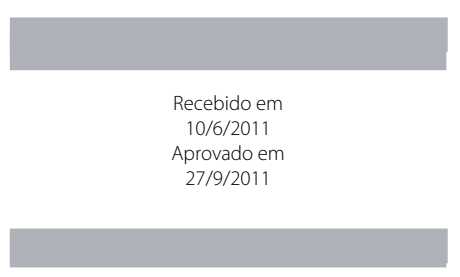

1 Universidade de São Paulo (USP), Faculdade de Medicina, Instituto de Psiquiatria (IPq), Hospital das Clínicas, Grupo Interdisciplinar de Estudos em Álcool e Drogas (GREA).

2 Universidade Federal de São Paulo (Unifesp), Faculdade de Medicina, Departamento de Ginecologia, Laboratório de Ginecologia Molecular. 3 USP, Faculdade de Medicina, Departamento de Psiquiatria, IPq, Hospital das Clínicas, Serviço de Psiquiatria da Infância e Adolescência (SEPIA).

Endereço para correspondência: Guilherme Rubino de Azevedo Focchi Rua Dr. Ovídio Pires de Campos, 785, Cerqueira César - 05403-903 - São Paulo, SP Telefone: (11) 2661-6960

E-mail grfocchi@gmail.com 


\section{INTRODUÇÃO}

Atualmente, há cerca de 1,3 bilhão de fumantes no mundo, e a metade deles, se continuar fumando, morrerá prematuramente de doenças cardiovasculares, câncer ou doenças respiratórias. A expectativa de vida dos fumantes é em média $25 \%$ menor que a dos não fumantes. O cigarro causa cerca de 5 milhões de mortes anualmente no mundo e, se a tendência for mantida, o número de mortes em 2020 aumentará para 10 milhões ${ }^{1-5}$. No Brasil, 35\% dos homens e 27\% das mulheres fumam, totalizando cerca de 35 milhões de fumantes, sendo que no país ocorrem cerca de 200 mil mortes ao ano associadas ao uso de cigarros ${ }^{6}$. Nos Estados Unidos, $60 \%$ dos gastos com saúde são empregados no tratamento de patologias relacionadas ao tabagismo, totalizando 1 bilhão de dólares ao dia; no Brasil, os gastos com doenças relacionadas ao tabagismo ultrapassam 330 milhões de reais ao ano ${ }^{4,6}$. Pode-se afirmar que a dependência de nicotina constitui hoje a principal causa de óbito passível de prevenção nos países ocidentais, sendo um enorme problema de saúde pública. Isso reforça a necessidade de investimento em pesquisas sobre seus determinantes e de novas abordagens terapêuticas 7,8 .

Os tratamentos atualmente usados na dependência de nicotina são: a terapia cognitivo-comportamental, a terapia de reposição de nicotina (TRN) e os tratamentos farmacológicos com bupropiona, nortriptilina e, mais recentemente, com vareniclina9,10. No caso específico da bupropiona, as taxas de cessação do uso de cigarro variam de $25 \%$ a $35 \%$ por um período de seis meses, altas se comparadas a outras medicações ${ }^{11}$.

Sabe-se que o comportamento de fumar é influenciado pelo genótipo, de forma que há efeito do polimorfismo DRD2 Taq1A na iniciação do tabagismo e da redução da atividade do polimorfismo CYP 2A6 no aumento do consumo de cigarros ${ }^{8,10}$. Nesse sentido, evidências em modelos humanos e animais sugerem a importância da dopamina como substrato do circuito de recompensa na dependência de nicotina. O foco atual das pesquisas tem se voltado para a influência da variação individual na transmissão dopaminérgica na resposta aos tratamentos farmacológicos para tabagismo ${ }^{12}$.

A bupropiona liga-se ao transportador dopaminérgico; $\mathrm{O}$ polimorfismo SLC6A3 3'UTR VNTR aumenta a codificação do transportador e, assim, poderia facilitar a ação desse fármaco. A associação entre a presença desse polimorfismo e o emprego da bupropiona, que funciona como agonista dopaminérgico, aumentaria a disponibilidade de dopamina endógena, e consequentemente o comportamento de procura pela nicotina seria reduzido ${ }^{13}$, facilitando a cessação do uso do cigarro. A presença do alelo 9 do referido polimorfismo estaria relacionada à maior taxa de cessação de cigarros em comparação ao alelo 10, o que justificaria o seu estudo ${ }^{13}$.

Uma metanálise envolvendo cinco estudos, com um total de 2.155 sujeitos, investigou a presença do polimorfismo
SLC6A3 3'UTR VNTR e sua relação com a parada do uso de cigarros. Verificou-se que em quatro deles houve tendência, porém sem resultado significativo, à associação entre a presença desse polimorfismo e a cessação do uso de cigarros. Os autores enfatizam a importância de mais pesquisas sobre esse polimorfismo no tratamento de fumantes ${ }^{13}$.

No Brasil, não há estudos controlados farmacogenéticos sobre o referido polimorfismo e sua influência no tratamento de dependentes de nicotina com a bupropiona. Considerando que as diferentes etnias refletem diferenças genotípicas, faz-se necessário o estudo de polimorfismos na população brasileira, que é altamente miscigenada.

Dessa forma, o objetivo deste estudo é avaliar a relação entre a resposta ao tratamento psiquiátrico da dependência de nicotina e a presença do polimorfismo SLC6A3 3'UTR VNTR em uma amostra clínica brasileira.

\section{MÉTODOS}

\section{Seleção dos sujeitos}

Os pacientes foram acompanhados em ambulatório específico para o atendimento de dependentes de nicotina no Grupo Interdisciplinar de Estudos de Álcool e Drogas do Instituto de Psiquiatria do Hospital das Clínicas da Faculdade de Medicina da Universidade de São Paulo (GREA-IPQ-HCFMUSP), no período de agosto de 2008 a novembro de 2009.

Foram selecionados 100 sujeitos dependentes de nicotina, mediante divulgação da pesquisa dentro do Complexo HC e da FMUSP. Os critérios de inclusão foram: indivíduos de ascendência europeia, segundo critério do avaliador, para possibilitar a comparação dos resultados com outros estudos ${ }^{10}$; sexo masculino; idade entre 18 e 50 anos; diagnóstico de dependência de nicotina pelo Diagnostic and Statistical Manual of Mental Disorders, quarta edição (DSM-IV); ; uso de mais de 10 cigarros por dia há pelo menos um ano; residência fixa na grande São Paulo; primeiro grau completo; telefone para contato e assinatura do termo de consentimento livre e esclarecido ${ }^{14}$. Os critérios de exclusão foram: presença de patologias clínicas ou psiquiátricas que necessitassem de tratamento em regime de internação; patologias cardíacas, como arritmias e infarto agudo do miocárdio; comorbidades psiquiátricas; uso de medicação psiquiátrica; antecedentes de epilepsia e convulsões ${ }^{14}$ e hipersensibilidade à bupropiona. A presença de patologias psiquiátricas foi verificada por meio de entrevista psiquiátrica, realizada pelo médico psiquiatra e principal pesquisador do estudo, e que também foi o responsável pela seleção da amostra.

De início, foram triados 250 indivíduos; foram excluídos 150 sujeitos, que não obedeceram aos critérios de seleção, pois apresentavam outros diagnósticos (por exemplo: depressão, dependência alcoólica) e pelo uso de outras medi- 
cações (por exemplo: antidepressivos). Estes foram encaminhados para outros serviços de tratamento de dependência de nicotina.

O estudo foi aprovado pela Comissão de Ética para Análise de Projetos de Pesquisa (CAPPesq) da Diretoria Clínica do Hospital das Clínicas da Faculdade de Medicina da Universidade de São Paulo (protocolo no 798/05).

\section{Tratamento e seguimento}

Após avaliação dos critérios de inclusão e exclusão, a proposta e a metodologia do estudo foram explicadas detalhadamente a cada paciente, enfatizando-se a obrigatoriedade da coleta de sangue no início do tratamento e o uso da medicação.

Foi utilizado o antidepressivo bupropiona, combinado à terapia de grupo de modelo cognitivo-comportamental, por 12 semanas ${ }^{15,16}$. As consultas médicas foram realizadas uma vez por semana, com duração de 30 minutos, e os pacientes, no mesmo dia da consulta individual, foram atendidos em grupo de terapia cognitivo-comportamental, pelo mesmo médico.

Os grupos de terapia tiveram entre quatro e seis pacientes por grupo. As sessões de grupo tiveram duração de 50 minutos, enfocando a prevenção de recaída no tabagismo, como a identificação dos fatores de recaída e estratégias para lidar com a "fissura" pelo cigarro. Os temas nos grupos não foram definidos previamente, e o terapeuta procurou coordenar as discussões, sempre enfocando a questão da dependência de nicotina e a prevenção de recaídas.

Na primeira consulta individual, os pacientes receberam bupropiona na dose de $150 \mathrm{mg}$ (1 cp) ao dia nos primeiros quatro dias, aumentando a dose após esse período para $300 \mathrm{mg}$ ao dia ( 2 cps, sendo um de manhã e um à tarde). A bupropiona $300 \mathrm{mg}$ ao dia foi mantida até o final do tratamento, sendo então retirada abruptamente ${ }^{17}$. Os pacientes poderiam interromper a medicação caso apresentassem efeitos colaterais intoleráveis. O controle da medicação e de seu fornecimento aos pacientes foi realizado pelo médico, que dava aos pacientes comprimidos em quantidade suficiente até o retorno seguinte. Os efeitos colaterais pela medicação foram coletados durante as consultas individuais, pelo médico. Não foi empregado questionário para checagem dos efeitos colaterais, para evitar indução de relato pelos pacientes, dando-se, assim, liberdade para que estes observassem espontaneamente as reações adversas, quando ocorressem. Os pacientes que abandonaram o tratamento não foram repostos, sendo também considerados na avaliação dos resultados desta intervenção.

A Escala de Fagerström, questionário que avalia a gravidade da dependência de nicotina, foi aplicada na primeira e na última consulta individual18,19.

Os dados sociodemográficos e o perfil de consumo de cigarros foram coletados na anamnese, pelo médico. No caso, a anamnese foi realizada mediante entrevista semiestruturada, com as questões focadas no tema tabagismo.

Na primeira consulta, foram colhidos $10 \mathrm{ml}$ de sangue de cada paciente (para evitar perdas de material) e, em seguida, armazenados para estudo genético.

\section{Estudo genético}

Após a extração do DNA, foi realizada a amplificação por meio da reação de cadeia de polimerase (PCR). As sequências de "primer" utilizadas para a amplificação do DNA foram 5' - TGT GGT GTA GGG AAC GGC CTG AG - 3' e 5' - CTT CCT GGA GGT CAC GGC TCA AGG - 3' (alelo 9 do polimorfismo SLC6A3 3'UTR VNTR) ${ }^{20}$.

\section{Seguimento pós-estudo}

Um mês após o final do tratamento, os pacientes foram avaliados uma única vez por telefone, no sentido de averiguar se estavam ou não abstinentes de nicotina ${ }^{9}$. Foi perguntado aos que pararam de fumar se mantinham a cessação do uso de cigarro e aos que não pararam se haviam conseguido parar. Em ambos os casos, registrou-se resposta do tipo "sim" ou "não". O contato foi realizado pelo pesquisador, que lembrou os pacientes a respeito do telefonema na última semana de tratamento.

\section{Análise estatística}

Os dados sociodemográficos (estado civil, trabalho e escolaridade) foram analisados para a amostra completa. As pontuações na Escala de Fagerström, no início e no final do tratamento, foram comparadas. Ao final do estudo, os pacientes foram divididos em dois grupos: aqueles que cessaram o uso de cigarro e os que não cessaram seu uso. Os dois grupos foram comparados quanto à presença do polimorfismo SLC6A3 3'UTR VNTR, tentativas anteriores de parar de fumar, assiduidade no tratamento e número de cigarros fumados ao dia. Foi realizada análise bivariada (teste do qui-quadrado para variáveis categóricas e prova de Mann-Whitney para variáveis contínuas). Para avaliar a diferença na pontuação da Escala de Fagerström, foi empregado o teste de Wilcoxon. O nível de significância considerado foi de 5\%.

\section{RESULTADOS}

\section{Características sociodemográficas e padrão inicial de uso de nicotina}

Na amostra total, $64 \%$ dos pacientes $(n=64)$ eram casados, 24\% ( $n=24)$, solteiros, $11 \%(n=11)$, separados e 1\% (1 paciente), viúvo. A maioria dos pacientes (97\%, ou 97 pacientes) estava trabalhando; quanto à escolaridade, $67 \%(n=67)$ tinham segundo grau completo, 28\% $(n=28)$ tinham terceiro grau completo e apenas $5 \%(n=5)$ tinham primeiro grau completo. 
A média/desvio-padrão (DP) da pontuação da Escala de Fagerström no início do tratamento foi de 5,96 +/- 2,14. A média/DP do número de cigarros fumados ao dia no início do estudo foi de $31,85+/-14,52$.

\section{Aderência ao tratamento e cessação do uso de cigarro}

Dos 100 pacientes que iniciaram o estudo, 80\% $(n=80)$ se mantiveram até o final do tratamento. Conseguiu-se um total de $84 \%$ amostras de sangue $(n=84)$. Seis pacientes que colheram a amostra de sangue no início do tratamento abandonaram o estudo. Dos pacientes que permaneceram no estudo, 81\% ( $n=65)$ interromperam o uso do cigarro.

Considerando apenas os pacientes que completaram o estudo ( $n=80$ ), a média/DP da Escala de Fagerström no início do tratamento foi de 5,96+/-2,14, e, no final do tratamento, de $0,43+/-1,25$, encontrando-se redução significativa na gravidade de dependência segundo esse instrumento $(p<0,0001)$.

\section{Análise da associação entre cessação de uso de cigarro versus polimorfismo}

Para o estudo da associação da cessação do uso e polimorfismo, considerou-se a amostra de 84 pacientes, que foram aqueles que foram genotipados.

Observa-se, na tabela 1, que 75\% $(n=63)$ cessaram o uso de cigarro e que $42,9 \%(n=36)$ tinham o polimorfismo, ao passo que 32,1\% ( $n=27)$ não tinham o polimorfismo. Quando foi analisada a cessação um mês após o final do tratamento, 41,6\% ( $n=35)$ dos pacientes pararam de fumar e apresentavam o polimorfismo, enquanto $28,6 \%(n=24)$ não o apresentavam (Tabela 2). Nessas situações, as diferenças entre a cessação do uso de cigarro e a presença do polimorfismo não foram significativas.

Tabela 1. Associação entre cessação do uso de cigarro e presença do polimorfismo SLC6A3 3'UTR VNTR no final do estudo

\begin{tabular}{lcccc}
\hline & \multicolumn{3}{c}{ Cessação no final do estudo } & \multirow{2}{*}{ p } \\
\cline { 2 - 4 } & Sim & Não & Abandonou & \\
\hline Presença de polimorfismo & $42,9 \%(36)$ & $7,1 \%(6)$ & $4,8 \%(4)$ & 0,44 \\
Ausência de polimorfismo & $32,1 \%(27)$ & $10,7 \%(9)$ & $2,4 \%(2)$ & \\
N Total & & 84 & & \\
\hline
\end{tabular}

Tabela 2. Associação entre cessação do uso de cigarro e presença do polimorfismo SLC6A3 3'UTR VNTR um mês após o final do tratamento

\begin{tabular}{lcccc}
\hline & \multicolumn{3}{c}{ Cessação após 1 mês } & \multirow{2}{*}{ p } \\
\cline { 2 - 4 } & Sim & Não & Abandonou & \\
\hline Presença de polimorfismo & $41,6 \%(35)$ & $8,3 \%(7)$ & $4,8 \%(4)$ & 0,19 \\
Ausência de polimorfismo & $28,6 \%(24)$ & $14,3 \%(12)$ & $2,4 \%(2)$ & \\
N total & & 84 & & \\
\hline
\end{tabular}

\section{Associação entre cessação do uso de cigarros, assiduidade no tratamento e número de cigarros fumados}

Não foi encontrada associação entre cessar o uso de cigarro ao término do tratamento, tentativas anteriores de parar de fumar e assiduidade no tratamento entre os pacientes que permaneceram no estudo (Tabela 3). Porém, com relação ao número de cigarros consumidos, verificou-se tendência de diferença significativa: a média/DP do número de cigarros consumidos foi menor $(30,15+/-13,4)$ entre aqueles que cessaram o uso de cigarro ao término do tratamento $(p=$ 0,06). Quando se considerou o período de um mês após o final do tratamento, quatro sujeitos que antes haviam parado de fumar retomaram o uso de cigarro. Não houve associação entre essas variáveis e a cessação do uso de cigarros (Tabela 4).

Tabela 3. Associação entre parada do uso de cigarros ao término do tratamento e número de cigarros fumados, tentativas anteriores de parar de fumar e assiduidade durante o tratamento

\begin{tabular}{|c|c|c|c|c|c|}
\hline & \multirow{2}{*}{$\begin{array}{l}\text { Cigarros } \\
\text { (Média/DP) }\end{array}$} & \multicolumn{2}{|c|}{$\begin{array}{c}\text { Tentativas anteriores de } \\
\text { parar de fumar }\end{array}$} & \multicolumn{2}{|c|}{ Assiduidade } \\
\hline & & Sim & Não & Sim & Não \\
\hline $\begin{array}{l}\text { Cessação do } \\
\text { uso ao final } \\
\text { do estudo } \\
(n=65)\end{array}$ & $30,15(13,4)$ & $93,8 \%(61)$ & $6,2 \%(4)$ & $35,4 \%(23)$ & $64,6 \%(42)$ \\
\hline $\begin{array}{l}\text { Não cessação } \\
\text { do uso ao final } \\
\text { do estudo } \\
(n=15)\end{array}$ & $37,67(15,2)$ & $100,0 \%(15)$ & $0,0 \%(0)$ & $13,3 \%(2)$ & $86,7 \%(13)$ \\
\hline$P$ & 0,060 & 0,3 & & & \\
\hline $\mathrm{N}$ total & & & 80 & & \\
\hline
\end{tabular}

Tabela 4. Associação entre parada do uso de cigarros um mês após o tratamento e número de cigarros fumados, tentativas anteriores de parar de fumar e assiduidade durante o tratamento

\begin{tabular}{|c|c|c|c|c|c|}
\hline & \multirow{2}{*}{$\begin{array}{c}\text { Cigarros } \\
\text { Média/(DP) }\end{array}$} & \multicolumn{2}{|c|}{$\begin{array}{c}\text { Tentativas anteriores de } \\
\text { parar de fumar }\end{array}$} & \multicolumn{2}{|c|}{ Assiduidade } \\
\hline & & Sim & Não & Sim & Não \\
\hline $\begin{array}{l}\text { Cessação } \\
\text { após } 1 \text { mês de } \\
\text { tratamento } \\
(n=59)\end{array}$ & $30,66(13,6)$ & $93,4 \%(57)$ & $6,6 \%(4)$ & $34,4 \%$ (21) & $65,6 \%(40)$ \\
\hline $\begin{array}{l}\text { Não cessação } \\
\text { após } 1 \text { mês de } \\
\text { tratamento } \\
(\mathrm{n}=21)\end{array}$ & $34,47(14,9)$ & $100,0 \%$ (19) & $0,0 \%(0)$ & $21,1 \%(4)$ & $78,9 \%(15)$ \\
\hline$P$ & 0,301 & \multicolumn{2}{|c|}{0,252} & \multicolumn{2}{|c|}{0,272} \\
\hline $\mathrm{N}$ total & & & 80 & & \\
\hline
\end{tabular}




\section{Efeitos colaterais}

O efeito colateral mais importante nos pacientes que permaneceram no estudo foi insônia (25\%), seguida de obstipação intestinal (15\%) e gosto amargo na boca (13,7\%). Um paciente relatou insônia grave e suspendeu a medicação, permanecendo, no entanto, até o final do tratamento. Esse paciente solicitou permanecer na terapia cognitivo-comportamental, foi incluído na análise genética e não apresentou o polimorfismo. Não houve relato de efeitos colaterais mais graves, como convulsões.

\section{DISCUSSÃO}

Foi verificada associação entre número de cigarros consumidos por dia e êxito na cessação do consumo destes: os pacientes que consumiam menos cigarros por dia tenderam a conseguir maior êxito na cessação do consumo. Isso evidencia o número de cigarros fumados ao dia como importante critério de gravidade, usado, inclusive, na Escala de Fagerström ${ }^{19}$. A intervenção empregada proporcionou a queda da pontuação nessa escala durante o tratamento, com diferença significativa entre o início do estudo e seu término. Isso mostra que o tratamento empregado foi efetivo para reduzir a gravidade da dependência. De fato, no tratamento do tabagismo, a simples redução dos sintomas da dependência já é benéfica para a saúde do indivíduo e pode contribuir para a cessação do tabagismo em tentativas posteriores de parar o cigarro. A parada total do uso de cigarros é o objetivo final do tratamento, e o tratamento precoce é fundamental, antes que o número de cigarros fumados ao dia aumente ${ }^{21}$.

Não foi verificada associação entre presença do alelo 9 do polimorfismo SLC6A3 3'UTR VNTR e a parada do uso de cigarros ao final das 12 semanas de tratamento, o que difere do verificado em outros trabalhos ${ }^{12,13}$. Um estudo duplo-cego randomizado controlado ${ }^{10}$, com 291 pacientes de ascendência europeia, que receberam bupropiona ou placebo, combinados à terapia de modelo cognitivo-comportamental por 12 semanas, fez a genotipagem para o receptor dopaminérgico D2 (DRD2-Taq1A), para o transportador de dopamina (SLC6A3 3'UTR VNTR) e para o citocromo CYP2B6. A dosagem de cotinina, marcador bastante confiável na averiguação da abstinência de nicotina, foi realizada durante o tratamento 9 . Nesse estudo, encontrou-se associação significativa entre o polimorfismo DRD2-Taq1A2A2 e a abstinência de nicotina com a bupropiona, havendo também interação estatisticamente significativa entre os polimorfismos DRD2 Taq1A e CYP2B6, de modo que o encontro desses dois genótipos correspondeu a maiores taxas de abstinência entre os participantes do estudo. Os autores fazem uma crítica ao número pequeno de pacientes, no referente à investigação farmacogenética. Entretanto, deve-se salientar o uso da do- sagem de cotinina e a importância do estudo da interação entre polimorfismos, no referido trabalho. A diferença com o presente estudo pode ser decorrente do tamanho menor da amostra, que teria sido insuficiente para identificar associação. Dessa forma, evidências indicam efeito do polimorfismo do sistema dopaminérgico DRD2Taq1 A2A2 e da interação dos polimorfismos DRD2Taq1A e CYP2B6 no processo de facilitação da abstinência de cigarros 22,23 .

Outro estudo, com 583 pacientes tabagistas, examinou a associação entre o polimorfismo SLC6A3 3'UTR VNTR e o tratamento da dependência de nicotina com terapia de reposição de nicotina ou bupropiona (à escolha do paciente) e terapia cognitivo-comportamental, por 12 semanas. Nesse caso, encontrou-se efeito positivo do polimorfismo na parada precoce de cigarros $^{24}$. O número de pacientes nesse estudo é maior, porém o fato de os pacientes escolherem o tratamento medicamentoso criaria um viés no sentido de saber qual a medicação realmente efetiva na cessação do cigarro.

De fato, a presença do polimorfismo pode não ser o único fator a se relacionar com a resposta ao tratamento. O tamanho do polimorfismo (número de "repeats") poderia também contribuir para processos dinâmicos que regulariam a densidade do transportador de dopamina, incluindo sua expressão proteica. Nesse caso, não é possível calcular o equilíbrio de Hardy-Weinberg $\left({ }^{13,25}\right)$. A presença do alelo 9 do polimorfismo SLC6A3 3'UTR VNTR poderia reduzir a recaptação de dopamina, facilitando a cessação de cigarros pelo paciente, pois haveria maior disponibilidade desse neurotransmissor no circuito de recompensa cerebral ${ }^{13,24}$. Além disso, deve-se salientar que outros polimorfismos poderiam atuar em conjunto ${ }^{10,13}$. O polimorfismo SLC6A3 3'UTR VNTR poderia também interagir com adaptações neuropsicológicas persistentes resultantes do tratamento, podendo auxiliar na manutenção da abstinência ${ }^{10}$.

Não houve problemas de tolerância à medicação, sendo a frequência de efeitos colaterais similar à encontrada em outros trabalhos, ressaltando-se a segurança da bupropiona no tratamento da dependência de nicotina ${ }^{11,15}$.

O presente estudo apresenta algumas limitações. A amostra estudada foi limitada em 100 pacientes, um número considerado pequeno para estudos genéticos ${ }^{10}$. Porém, é importante lembrar que esse estudo era também de resposta ao emprego de medicação no tratamento de dependência, ou seja, um estudo clínico que apresenta dificuldades para ser realizado com amostras maiores, tais como a seleção da amostra segundo os critérios bastante específicos de inclusão e exclusão. Nesse sentido, outra limitação é o critério de seleção de indivíduos de ascendência europeia a critério do examinador, a saber, pela cor da pele (branca). A ascendência não está apenas relacionada à cor de pele, sendo influenciada pelo genótipo individual, não avaliado neste trabalho. Além disso, é questionável a seleção de indivíduos de ascendência europeia na população brasileira, que 
tem alto grau de miscigenação. Outro aspecto importante a ser considerado foi a não utilização de instrumentos de avaliação para a triagem de pacientes no sentido de excluir comorbidades psiquiátricas. Optou-se pela entrevista clínica como instrumento de triagem, porque muitos indivíduos poderiam apresentar sintomas ansiosos ou depressivos, não configurando, porém, diagnóstico psiquiátrico. Entretanto, estudos futuros poderão empregar instrumentos para avaliar a intensidade de sintomas e correlacioná-los com a resposta ao tratamento. Da mesma forma, a avaliação do grau de motivação dos pacientes para o tratamento poderia ser realizada, no sentido de homogeneizar os grupos de tratamento e reduzir, assim, a possibilidade de abandonos.

Outro aspecto que merece discussão é o uso de dispositivos de nicotina, como goma e adesivo, que poderiam ser usados como forma de tornar a terapêutica mais abrangente. Porém, o seu uso neste trabalho criaria um viés, na medida em que não se saberia até que ponto a parada do uso de cigarros poderia ser atribuída ao efeito específico da bupropiona.

Também, não foram analisados outros polimorfismos que poderiam interagir mutuamente e que poderiam estar associados de forma significativa à parada do uso de cigarros $^{10}$. O citocromo CYP2B6, por exemplo, metaboliza a nicotina, reduzindo seu nível sérico e reforçando, assim, a procura por cigarros ${ }^{26}$.

Por fim, outro aspecto a ser considerado é o tempo de seguimento dos pacientes. Neste trabalho, o tempo de seguimento foi relativamente curto (12 semanas, mais um telefonema um mês após o término do tratamento), e a maioria dos trabalhos publicados sobre o tema faz o follow-up de 12 meses, haja vista o alto grau de recaídas a médio e longo prazo ${ }^{14,22}$. Entretanto, um tempo de seguimento mais longo aumentaria a possibilidade de outros fatores (por exemplo: sociais) interferirem no estudo, criando um viés e dificultando encontrar a associação entre o polimorfismo e a cessação do tabagismo.

Apesar das limitações discutidas acima, este trabalho é o primeiro sobre psicofarmacogenética no tratamento psiquiátrico do tabagismo no Brasil, país com alto grau de miscigenação de sua população. Este estudo cria possibilidades futuras de desenvolvimento de outros trabalhos abordando a psicofarmacogenética do tabagismo e que contem com o emprego de metodologias mais refinadas e maior número de pacientes, tendo como objetivo a seleção de amostras de indivíduos que responderiam mais efetivamente a tratamentos propostos, com um mínimo de efeitos colaterais.

\section{CONCLUSÃO}

O maior entendimento dos mecanismos genéticos da dependência de nicotina oferece a possibilidade de tratamento personalizado aos pacientes, baseado no genótipo. Isso se configura como o futuro da medicina: o tratamento far- macogenético, individualizado, com a seleção de pacientes baseada em seu perfil gênico, levaria à maior efetividade do tratamento proposto, com um mínimo de efeitos colaterais. No caso da dependência de nicotina, deve-se salientar a necessidade de terapias combinadas - medicamentosa e cognitivo-comportamental -, pois é necessária a mudança do estilo de vida por parte do paciente para que este se mantenha abstinente de cigarros ${ }^{10,24}$. Este trabalho aponta para a necessidade de mais estudos, que contem com melhorias metodológicas, para avaliar se a presença do polimorfismo SLC6A3 3'UTR VNTR poderia melhorar a resposta ao tratamento da dependência de nicotina, sendo importante como modelo para outros estudos futuros.

\section{AGRADECIMENTOS}

À Maria Rita Passos Bueno, do Instituto de Genômica da Universidade de São Paulo (USP), e à Cristiane Alves Oliveira, do Laboratório de Ginecologia Molecular da Universidade Federal de São Paulo (Unifesp).

O presente trabalho recebeu apoio financeiro (auxílio-pesquisa) da Fundação de Amparo à Pesquisa do Estado de São Paulo (Fapesp) (projeto 07-54410-4).

\section{REFERÊNCIAS}

1. Centers for Disease Control and Prevention. Cigarette smoking - atributable mortality and years of potential life lost, United States. MMWR. 1990;42:645-9.

2. Peto R, Lopez AD, Boreham J, Thun M, Heath C Jr. Mortality from smoking in developed countries 1950-2000. New York: Oxford University Press; 1994.

3. APA - American Psychiatric Association. Practice guideline for the treatment of patients with nicotine dependence. Am J Psychiatry. 1996;151:1-31.

4. Shafey 0, Dolwick S, Guindon GE. Tobacco control country profiles 2003. Atlanta: American Cancer Society; 2003.

5. Ezzati M, Lopez AD. Estimates of global mortality attributable to smoking in 2000. Lancet. 2003:362:847-52.

6. Tobacco Control Country Profiles. American Cancer Society; 1993.

7. APA - American Psychiatric Association. Diagnostic and Statistical Manual of Mental Disorders. Washington: American Psychiatric Press; 1994.

8. Li MD. The genetics of smoking cessation: a brief review. Am J Med Sci. 2003;326:168-73.

9. Orleans CT, Slade J. Nicotine addiction: principles and management. Oxford: Oxford University Press; 1993

10. David S, Brown RA, Papandonatos GD, Kahler CW, Lloyd-Richardson EE, Munafò MR, et al. Pharmacogenetic clinical trial of sustained release bupropion for smoking cessation. Nicotine Tob Res. 2007;9(8):821-33.

11. Hurt RD, Sachs SP, Glover ED, Offord KP, Johnston JA, Dale LC, et al. A comparison of sustained-release bupropion and placebo for smoking cessation. N Engl J Med. 1997;337:1195-202.

12. Lerman C, Niaura R. Applying genetic approaches to the treatment of nicotine dependence. Oncogene. 2002;21:7412-20.

13. Stapleton JA, Sutherland G, O'Gara C. Association between dopamine transporter genotypes and smoking cessation: a meta-analysis. Addict Biol. 2007;12:221-6.

14. David S, Niaura B, Papandonatos G, Shadel W, Burkholder G, Britt D, et al. Does the DRD2 Taq 1 a polimorphism influence treatment response to bupropion hydrocloride for reduction of the nicotine withdrawal syndrome? Nicotine Tob Res. 2003;5:935-42. 
15. Holm JH, Spencer CM. Bupropion: a review of its use in the management of smoking cessation. Drugs. 2000;59:135-50.

16. Sims TH, Fiore MC. Pharmacotherapy for treating tobacco dependence. What is the ideal duration of therapy? CNS Drugs. 2002;16:653-62.

17. Ahluwalia IS, Harris KJ, Catley D, Okuyemi KS, Mayo MS. Sustained release bupropion for smoking cessation in African Americans. JAMA. 2002;288:468-73.

18. Fagerström KO, Schneider NG. Measuring nicotine dependence: a review of the Fagerström Tolerance Questionnaire. J Behav Med. 1989;12:159-81.

19. Heatherton TF, Koslowski LT, Frecker RC. The Fagerström Test for Nicotine Dependence: a revision of the Fagerström Tolerance Questionnaire. Br J Addict. 1991;86:1119-27.

20. Vandenbergh DJ, Persico AM, Hawkins AL, Griffin CA, LiX, Jabs EW, et al. Human dopamine transporter gene (DAT1) maps to chromosome 5p.15.3 and displays a VNTR. Genomics. 1992;14:114-6.
21. Scivoletto S. Tabagismo em adolescentes. In: Focchi GRA, Malbergier A, Ferreira MP, editores. Tabagismo: dos fundamentos ao tratamento. São Paulo: Lemos; 2006. p. 143-55.

22. Munafò MR, Clark TG, Johnstone E, Murphy MFG, Walton RT. The genetic basis for smoking behavior: a systematic review and meta-analysis. Nicotine Tob Res. 2004;6(4):583-98.

23. Swan GE, Valdes AM, Ring HZ. Dopamine receptor DRD2 genotype and smoking cessation outcome following treatment with Bupropion SR. Pharmacogenomics J. 2005;5:21-9.

24. O'Gara C, Stapleton JA, Sutherland G, Guindalini C, Neale B, Breen G, et al. Dopamine transporter polymorphisms are associated with short term response to smoking cessation treatment. Pharmacogenet Genomics. 2007;17:61-7.

25. Miller GM, Madras BK. Polymorphisms in the $3^{\prime}$ untranslated region of human and monkey dopamine transporter genes affect reporter gene expression. Mol Psychiatry. 2002;7:44-55.

26. Yamanaka H, Nakajima N, Fukami T. CYP2A6 and CYP2B6 are involved in nornicotine formation from nicotine in humans: interindividual differences in these contributions. Drug Metab Dispos. 2005;33:1811-8. 